\title{
The First Transmission Electron Microscope Image Imagined by Artificial Intelligence
}

\author{
Jongyeong Lee, Zonghoon Lee* \\ School of Materials Science and Engineering, Ulsan National Institute of Science and Technology (UNIST), UIsan 44919, Korea
}

*Correspondence to:

Lee Z,

Tel: +82-52-217-2327

Fax: +82-52-217-2309

E-mail: zhlee@unist.ac.kr

Received December 11, 2017

Revised December 14, 2017

Accepted December 14, 2017

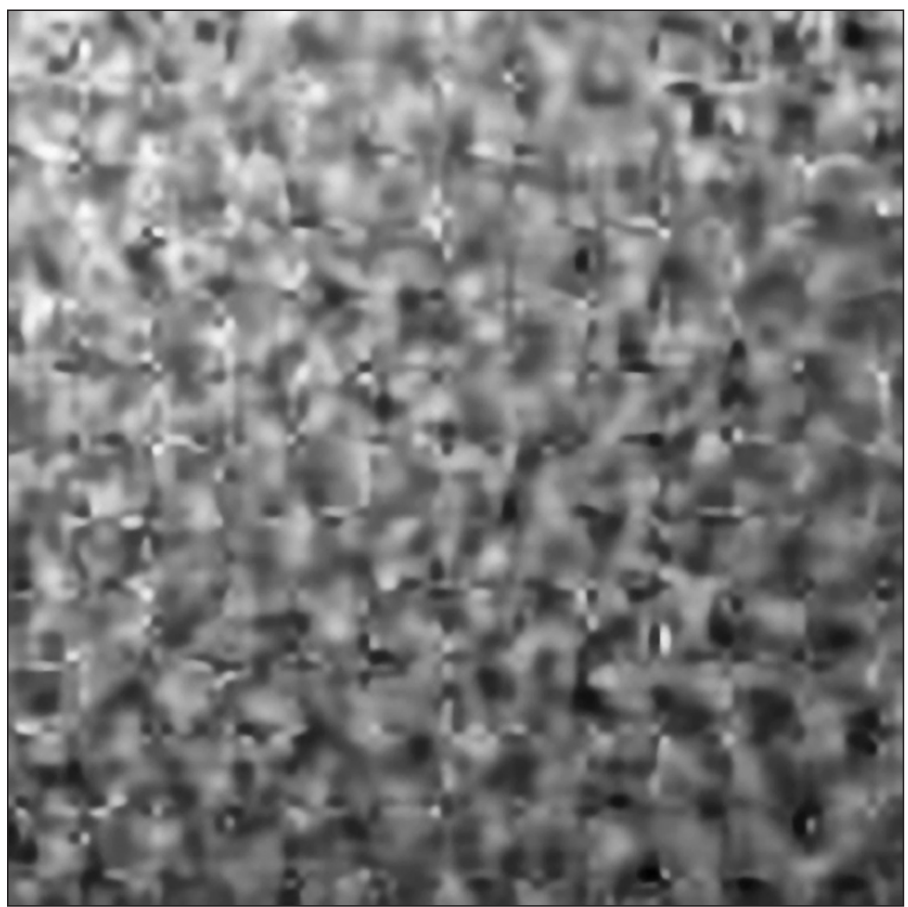

Generative Adversarial Networks (GANs) are a class of Artificial Intelligence that consist of a generative neural network and a discriminative neural network (Goodfellow et al., 2014). The generative neural network learns to imagine fake images, and the discriminative neural network learns to distinguish between "real" and "fake" images. Learning enables the discriminative neural network to discriminate more effectively, while enabling the generative neural network to imagine (or generate) concrete shapes. The fake images, created by the generative neural network, contain characteristics of the real images that were used as the input data for the GAN. In this study, we used atomic resolution graphene images as input data, obtained by an aberrationcorrected FEI Titan Cubed TEM (FEI Titan ${ }^{3}$ G2 60-300) to train this GAN. This image is the output of the GAN imagined by the generative neural network. It has a contrast and signal to noise ratio similar to the original image. It is also believed to include the physical and structural characteristics of the graphene image input by the interpretable representation learning study (Chen et al., 2016). Furthermore, this technology can be used as a criterion for neural network's comprehension about transmission electron microscopy.

[The 3rd East-Asia Microscopy Conference, Novus Imago Best Technical Award]

(a) This is an open-access article distributed under the terms of the Creative Commons Attribution Non-Commercial License (http://creativecommons.org/licenses/by-nc/4.0) which permits unrestricted noncommercial use, distribution, and reproduction in any medium, provided the original work is properly cited.

Copyrights @ 2017 by Korean Society of Microscopy 


\section{CONFLICT OF INTEREST}

No potential conflict of interest relevant to this article was reported.

\section{REFERENCES}

Goodfellow I, Pouget-Abadie J, Mirza M, Xu B, Warde-Farley D, Ozair S, Courville A, and Bengio Y (2014) Generative adversarial nets. In: Advances in Neural Information Processing Systems 27 (NIPS 2014), pp. 2672-2680, (NIPS, Montréal).
Chen X, Duan Y, Houthooft R, Schulman J, Sutskever I, and Abbeel P (2016) InfoGAN: interpretable representation learning by information maximizing generative adversarial nets. In: Advances in Neural Information Processing Systems 29 (NIPS 2016), pp. 2172-2180, (NIPS, Barcelona). 